\title{
Completeness of Combinations of Constructor Systems ${ }^{\dagger}$
}

\author{
Aart Middeldorp ${ }^{\ddagger}$ \\ Centre for Mathematics and Computer Science, \\ Kruislaan 413, 1098 SJ Amsterdam, The Netherlands. \\ email: ami @cwi.nl \\ Yoshihito Toyama \\ Centre for Mathematics and Computer Science, \\ Kruislaan 413, 1098 SJ Amsterdam, The Netherlands; \\ NTT Basic Research Laboratories \\ 3-9-11 Midori-cho, Musashino-shi, Tokyo 180, Japan. \\ email: toyama @cwi.nl
}

\begin{abstract}
A term rewriting system is called complete if it is both confluent and strongly normalizing. Barendregt and Klop showed that the disjoint union of complete term rewriting systems does not need to be complete. In other words, completeness is not a modular property of term rewriting systems. Toyama, Klop and Barendregt showed that completeness is a modular property of left-linear TRS's. In this paper we show that it is sufficient to impose the constructor discipline for obtaining the modularity of completeness. This result is a simple consequence of a quite powerful divide and conquer technique for establishing completeness of such constructor systems. Our approach is not limited to systems which are composed of disjoint parts. The importance of our method is that we may decompose a given constructor system into parts which possibly share function symbols and rewrite rules in order to infer completeness. We obtain a similar technique for semi-completeness, i.e. the combination of confluence and weak normalization.
\end{abstract}

\section{Introduction}

A property of term rewriting systems is modular if it is preserved under disjoint union. Starting with Toyama [19], several authors studied modular aspects of term rewriting systems. Toyama [19] showed that confluence is a modular property. In [20] Toyama refuted the modularity of strong normalization by means of the following term rewriting systems:

$$
\begin{aligned}
& R_{1}=\{F(0,1, x) \rightarrow F(x, x, x)\} \\
& R_{2}=\left\{\begin{array}{l}
g(x, y) \rightarrow x \\
g(x, y) \rightarrow y .
\end{array}\right.
\end{aligned}
$$

Both systems are terminating, but their union admits the following cyclic reduction:

$\dagger$ This paper is an abbreviated version of CWI report CS-R9059.

¥ Partially supported by ESPRIT Basic Research Action 3020, INTEGRATION. 


$$
\begin{aligned}
F(g(0,1), g(0,1), g(0,1)) & \rightarrow F(0, g(0,1), g(0,1)) \\
& \rightarrow F(0,1, g(0,1)) \\
& \rightarrow F(g(0,1), g(0,1), g(0,1)) .
\end{aligned}
$$

His counterexample inspired Rusinowitch [18] to the formulation of sufficient conditions for the strong normalization of the disjoint union of strongly normalizing term rewriting systems $R_{1}$ and $R_{2}$ in terms of the distribution of collapsing and duplicating rules among $R_{1}$ and $R_{2}$. Rusinowitch's results were extended by Middeldorp [11]. Barendregt and Klop gave an example showing that completeness (i.e. the combination of confluence and strong normalization) is not a modular property, see Toyama [20]. Independently, Drosten [3] gave the following simpler counterexample:

$$
\begin{aligned}
& \mathcal{R}_{1}= \begin{cases}F(0,1, x) & \rightarrow F(x, x, x) \\
F(x, y, z) & \rightarrow 2 \\
0 & \rightarrow 2 \\
1 & \rightarrow 2\end{cases} \\
& \mathcal{R}_{2}=\left\{\begin{array}{lll}
g(x, y, y) & \rightarrow x \\
g(y, y, x) & \rightarrow x .
\end{array}\right.
\end{aligned}
$$

Both systems are easily shown to be complete. However, because both $g(0,1,1) \rightarrow 0$ and $g(0,1,1) \rightarrow 1$, the term $F(g(0,1,1), g(0,1,1), g(0,1,1))$ has a cyclic reduction akin to the one in the previous counterexample. Toyama, Klop and Barendregt [22] showed that the restriction to left-linear term rewriting systems is sufficient for obtaining the modularity of completeness. Middeldorp [10] showed that the property of having unique normal forms is modular for general term rewriting systems. An interesting alternative approach to modularity is explored in Kurihara and Kaji [7]. Middeldorp [12,13,14] extended the above results to conditional term rewriting systems. Kurihara and Ohuchi [8] showed that strong normalization is a modular property of term rewriting systems whose strong normalization can be shown by a simplification ordering. They extended this result in [9] to term rewriting systems which share constructors. Constructors are function symbols which do not occur at the leftmost position in left-hand sides of rewrite rules. Dershowitz [1], Geser [4] and Toyama [21] give further results on combinations of term rewriting systems with common function symbols. A comprehensive survey of combinations of (conditional) term rewriting systems can be found in Middeldorp [15].

The starting point of the present paper is the refutation of the modularity of completeness. We show that instead of requiring left-linearity it is also possible to impose the so-called constructor discipline for obtaining the modularity of completeness. In a constructor system (a term rewriting system which obeys the constructor discipline) all function symbols occurring at non-leftmost positions in left-hand sides of rewrite rules are constructors. Many term rewriting systems that occur in practice follow this discipline, see e.g. O'Donnell [17]. Actually we prove a much stronger result. We show that a constructor system is complete if it can be decomposed into complete constructor systems. The important observation is that our notion of decomposition does not imply disjointness. Consider for example the constructor system 


$$
\mathcal{R}= \begin{cases}0+x & \rightarrow x \\ S(x)+y & \rightarrow S(x+y) \\ 0 \times x & \rightarrow 0 \\ S(x) \times y & \rightarrow x \times y+y \\ f(0) & \rightarrow 0 \\ f(S(x)) & \rightarrow f(x)+S(x) .\end{cases}
$$

We can decompose $R$ into

$$
R_{1}=\left\{\begin{array}{ll}
0+x & \rightarrow x \\
S(x)+y & \rightarrow S(x+y) \\
0 \times x & \rightarrow 0 \\
S(x) \times y & \rightarrow x \times y+y
\end{array} \quad \text { and } \quad R_{2}=\left\{\begin{array}{l}
0+x \rightarrow x \\
S(x)+y \rightarrow S(x+y) \\
f(0) \\
f(S(x)) \rightarrow 0
\end{array}\right.\right.
$$

Both systems are easily shown to be complete and our decomposition result yields the completeness of $R$. Neither the result of Kurihara and Ohuchi [9] (because $R_{1}$ and $R_{2}$ share the non-constructor symbol + ) nor the result of Dershowitz [1] (because $R_{1}$ and $R_{2}$ are not right-linear) applies.

In the next section we give a concise introduction to term rewriting. Extensive surveys are Dershowitz and Jouannaud [2] and Klop [6]. In Section 2 we introduce the concept of marked reduction which plays a crucial role in the proof of our main results. Section 3 contains our main results. We define a notion of decomposability and we show that completeness is a decomposable property of constructor systems. To appreciate the non-triviality of our result, it may be contrasted with the fact that neither confluence nor strong normalization is decomposable. We further show that semi-completeness (i.e the combination of confluence and weak normalization) is a decomposable property of constructor systems.

\section{Preliminaries}

Let $\mathcal{V}$ be a countably infinite set of variables. A term rewriting system (TRS for short) is a pair $(\mathcal{F}, R)$. The set $\mathcal{F}$ consists of function symbols; associated to every $F \in \mathcal{F}$ is a natural number denoting its arity. Function symbols of arity 0 are called constants. The set $\mathcal{T}(\mathcal{F}, \mathcal{V})$ of terms built from $\mathcal{F}$ and $\mathcal{V}$ is the smallest set such that $\mathcal{V} \subset \mathcal{T}(\mathcal{F}, \mathcal{V})$ and if $F \in \mathcal{F}$ has arity $n$ and $t_{1}, \ldots, t_{n} \in \mathcal{T}(\mathcal{F}, \mathcal{V})$ then $F\left(t_{1}, \ldots, t_{n}\right) \in \mathcal{T}(\mathcal{F}, \mathcal{V})$. Identity of terms is denoted by $\equiv$. The root symbol of a term $t$ is defined as follows: $\operatorname{root}(t)=F$ if $t \equiv F\left(t_{1}, \ldots, t_{n}\right)$ and $\operatorname{root}(t)=t$ if $t \in \mathcal{V}$. The set $R$ consists of pairs $(l, r)$ with $l, r \in \mathcal{T}(\mathcal{F}, \mathcal{V})$ subject to the following two constraints:

(1) the left-hand side $l$ is not a variable,

(2) the variables which occur in the right-hand side $r$ also occur in $l$.

Pairs $(l, r)$ are called rewrite rules and will henceforth be written as $l \rightarrow r$. A rewrite rule $l \rightarrow r$ is left-linear if $l$ does not contain multiple occurrences of the same variable. A leftlinear TRS only contains left-linear rewrite rules.

A substitution $\sigma$ is a mapping from $\mathcal{V}$ to $\mathcal{T}(\mathcal{F}, \mathcal{V})$ such that its domain $\{x \in \mathcal{V} \mid \sigma(x) \not x\}$ is finite. Substitutions are extended to morphisms from $\mathcal{T}(\mathcal{F}, \mathcal{V})$ to $\mathcal{T}(\mathcal{F}, \mathcal{V})$, i.e. $\sigma\left(F\left(t_{1}, \ldots, t_{n}\right)\right) \equiv F\left(\sigma\left(t_{1}\right), \ldots, \sigma\left(t_{n}\right)\right)$ for every $n$-ary function symbol $F$ and terms $t_{1}, \ldots, t_{n}$. We call $\sigma(t)$ an instance of $t$. We write $t^{\sigma}$ instead of $\sigma(t)$. An instance of a left-hand side of a rewrite rule is a redex (reducible expression). Let $\square$ be a special constant 
symbol. A context $C[, \ldots$,$] is a term in \mathcal{T}(\mathcal{F} \cup\{\square\}, \mathcal{V})$. If $C[, \ldots$,$] is a context with n$ occurrences of $\square$ and $t_{1}, \ldots, t_{n}$ are terms then $C\left[t_{1}, \ldots, t_{n}\right]$ is the result of replacing from left to right the occurrences of $\square$ by $t_{1}, \ldots, t_{n}$. A context containing precisely one occurrence of $\square$ is denoted by $C$ [ ]. A term $s$ is a subterm of a term $t$ if there exists a context $C$ [ ] such that $t \equiv C[s]$. If $C[] \not \equiv \square$ then $s$ is a proper subterm of $t$. We write $s \subseteq t$ to indicate that $s$ is a subterm of $t$.

The rewrite relation $\rightarrow_{\mathscr{R}}$ is defined as follows: $s \rightarrow_{R} t$ if there exists a rewrite rule $l \rightarrow r$ in $R$ a substitution $\sigma$ and a context $C$ [ ] such that $s \equiv C\left[l^{\sigma}\right]$ and $t \equiv C\left[r^{\sigma}\right]$. The transitivereflexive closure of $\rightarrow_{R}$ is denoted by $\rightarrow_{R}$; if $s \rightarrow_{R} t$ we say that $s$ reduces to $t$. We write $s \leftarrow_{R} t$ if $t \rightarrow_{R} s$; likewise for $s \leftarrow_{R} t$. The transitive closure of $\rightarrow_{R}$ is denoted by $\rightarrow_{R}^{+}$and $\leftrightarrow_{\mathcal{R}}$ denotes the symmetric closure of $\rightarrow_{\mathcal{R}}$ (so $\leftrightarrow_{\mathcal{R}}=\rightarrow_{\mathcal{R}} \cup \leftarrow_{\mathcal{R}}$ ). The transitive-reflexive closure of $\leftrightarrow_{\mathcal{R}}$ is called conversion and denoted by $=_{\mathcal{R}}$. If $s=_{\mathcal{R}} t$ then $s$ and $t$ are convertible. Two terms $t_{1}, t_{2}$ are joinable, notation $t_{1} \downarrow_{\mathcal{R}} t_{2}$, if there exists a term $t_{3}$ such that $t_{1} \rightarrow_{R} t_{3} \leftarrow_{R} t_{2}$. Such a term $t_{3}$ is called a common reduct of $t_{1}$ and $t_{2}$. We often omit the subscript $R$.

A term $s$ is a normal form if there is no term $t$ with $s \rightarrow t$. A TRS is weakly normalizing if every term reduces to a normal form. A TRS is strongly normalizing if there are no infinite reduction sequences $t_{1} \rightarrow t_{2} \rightarrow t_{3} \rightarrow \ldots$. In other words, every reduction sequence eventually ends in a normal form. A TRS is confluent or has the Church-Rosser property if for all terms $s, t_{1}, t_{2}$ with $t_{1} \nleftarrow s \rightarrow t_{2}$ we have $t_{1} \downarrow t_{2}$. A well-known equivalent formulation of confluence is that every pair of convertible terms is joinable $\left(t_{1}=t_{2} \Rightarrow t_{1} \downarrow t_{2}\right)$. A TRS is locally confluent if for all terms $s, t_{1}, t_{2}$ with $t_{1} \leftarrow s \rightarrow t_{2}$ we have $t_{1} \downarrow t_{2}$. A complete TRS is confluent and strongly normalizing. A semi-complete TRS is confluent and weakly normalizing. These properties of TRS's specialize to terms in the obvious way. If a term $t$ has a unique normal form then we denote this normal form by $t \downarrow$.

The following. well-known result is due to Newman [16].

NEWMAN'S LEMMA. Every strongly normalizing and locally confluent TRS is confluent.

Let $l_{1} \rightarrow r_{1}$ and $l_{2} \rightarrow r_{2}$ be renamed versions of rewrite rules of a TRS $R$ such that they have no variables in common. Suppose $l_{1} \equiv C[t]$ with $t \notin \mathcal{V}$ such that $t$ and $l_{2}$ are unifiable, i.e. $t^{\sigma} \equiv l_{2}^{\sigma}$ for a most general unifier $\sigma$. The term $l_{1}^{\sigma} \equiv C\left[l_{2}\right]^{\sigma}$ is subject to the reduction steps $l_{1}^{\sigma} \rightarrow r_{1}^{\sigma}$ and $l_{1}^{\sigma} \rightarrow C\left[r_{2}\right]^{\sigma}$. The pair of reducts $\left\langle C\left[r_{2}\right]^{\sigma}, r_{1}^{\sigma}\right\rangle$ is a critical pair of $R$. If $l_{1} \rightarrow r_{1}$ and $l_{2} \rightarrow r_{2}$ are renamed versions of the same rewrite rule, we do not consider the case $C[] \equiv \square$. A critical pair $\langle s, t\rangle$ of a TRS $R$ is convergent if $s \downarrow_{\mathcal{R}} t$. The following lemma of Huet [5] expresses the significance of critical pairs.

CRITICAL PAIR LEMMA. A TRS $R$ is locally confluent if and only if all its critical pairs are convergent.

A constructor system (CS for short) is a TRS $(\mathcal{F}, \mathcal{R}$ ) with the property that $\mathcal{F}$ can be partitioned into disjoint sets $\mathcal{D}$ and $C$ such that every left-hand side $F\left(t_{1}, \ldots, t_{n}\right)$ of a rewrite rule of $R$ satisfies $F \in \mathcal{D}$ and $t_{1}, \ldots, t_{n} \in \mathcal{T}(\mathcal{C}, \mathcal{V})$. Function symbols in $\mathcal{D}$ are called defined symbols and those in $C$ constructors. To emphasize the partition of $\mathcal{F}$ into $\mathcal{D}$ and $C$ we write $(\mathcal{D}, C, \mathcal{R})$ instead of $(\mathcal{F}, \mathcal{R})$ and $\mathcal{T}(\mathcal{F}, \mathcal{V})$ is denoted by $\mathcal{T}(\mathcal{D}, C, \mathcal{V})$.

Since the behaviour of a Turing machine can be simulated by a CS (see Klop [6] for details), CS's have universal computing power. The restriction on the left-hand sides of rewrite rules of CS's enables a considerable simplification of many concepts and proofs. For 
instance, if $\langle s, t\rangle$ is a critical pair of a $\operatorname{CS}(\mathcal{D}, C, R)$ then there exist different rewrite rules $l_{1} \rightarrow r_{1}, l_{2} \rightarrow r_{2} \in R$ (with variables suitably renamed) and a most general unifier $\sigma$ of $l_{1}$ and $l_{2}$ such that $s \equiv r_{1}^{\sigma}$ and $t \equiv r_{2}^{\sigma}$.

\section{Marked Reduction}

In this section we introduce a new rewrite relation which plays an essential role in the proofs of our decomposition results. Due to lack of space no proofs are presented in this section. They can be found in the full version of the paper. Throughout this section we will be dealing with an arbitrary $\mathrm{CS}(\mathcal{D}, \mathcal{C}, \mathcal{R})$.

\section{DEFINITION 2.1.}

(1) The set $\mathcal{D}^{*}=\left\{F^{*} \mid F \in \mathcal{D}\right\}$ consists of marked defined symbols. Terms in $\mathcal{I}\left(\mathcal{D}^{*} \cup \mathcal{D}, C, \mathcal{V}\right)$ are called marked terms. An unmarked term belongs to $\mathcal{T}(\mathcal{D}, \mathcal{C}, \mathcal{V})$

(2) If $t$ is a marked term then $e(t) \in \mathcal{T}(\mathcal{D}, C, \mathcal{V})$ denotes the term obtained from $t$ by erasing all marks and $t^{*}$ denotes the term obtained from $t$ by marking every unmarked defined symbol in $t$.

(3) , Two marked terms $s$ and $t$ are similar, notation $s \approx t$, if $e(s) \equiv e(t)$. If $s$ and $t$ are similar then their intersection is the unique term $s \wedge t$ such that $s \wedge t \approx s \approx t$ and a defined symbol occurrence in $s \wedge t$ is marked if and only if the corresponding symbols in $s$ and $t$ are marked.

(4) The set $\mathcal{R}^{*}$ of marked rewrite rules is defined as $\left\{l^{*} \rightarrow r^{*} \mid l \rightarrow r \in R\right\}$.

EXAMPLE 2.2. Consider the $\operatorname{CS}\left(\mathcal{D}_{1}, C_{1}, \mathcal{R}_{1}\right)$ with $\mathcal{D}_{1}=\{F, G\}, C_{1}=\{S, 0\}$,

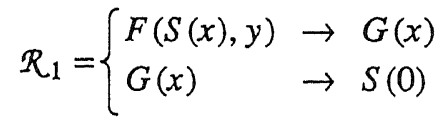

and the reduction sequence

$$
t \equiv F(S(G(0)), G(0)) \rightarrow F(S(G(0)), S(0)) \rightarrow G(G(0)) \rightarrow S(0) .
$$

If we mark some defined symbols in $t$ then we can easily mimic this sequence by a reduction sequence in $\mathcal{R}_{1} \cup \mathcal{R}_{1}^{*}$, for instance

$$
F^{*}\left(S\left(G^{*}(0)\right), G(0)\right) \rightarrow_{\mathbb{R}_{1}} F^{*}\left(S\left(G^{*}(0)\right), S(0)\right) \rightarrow_{\mathbb{R}_{i}^{*}} G^{*}\left(G^{*}(0)\right) \rightarrow_{\mathbb{R}_{i}^{*}} S(0) .
$$

This correspondence does not hold for non-left-linear CS's. Consider the CS $\left(\mathcal{D}_{2}, \mathcal{C}_{2}, R_{2}\right)$ with $\mathcal{D}_{2}=\{F\}, C_{2}=\{S\}, R_{2}=\{F(x, x) \rightarrow S(x)\}$ and the reduction step

$$
F(F(0, S(0)), F(0, S(0))) \rightarrow S(F(0, S(0))) \text {. }
$$

The marked term $F^{*}\left(F^{*}(0, S(0)), F(0, S(0))\right)$ cannot be reduced in $\mathcal{R}_{2} \cup \mathcal{R}_{2}^{*}$.

By modifying the rewrite relation associated to $R \cup \mathcal{R}^{*}$ we are able to mimic every unmarked reduction sequence, irrespective of the marking attached to the starting term.

DEFINTIION 2.3. We write $s \rightarrow_{m} t$ if there exists a context $C$ [ ], a rewrite rule

$$
C_{1}\left[x_{1}, \ldots, x_{n}\right] \rightarrow C_{2}\left[y_{1}, \ldots, y_{m}\right]
$$

in $R \cup \mathcal{R}^{*}$ (with all variables displayed) and terms $s_{1}, \ldots, s_{n}, t_{1}, \ldots, t_{m}$ such that the 
following three conditions are satisfied:

(1) $s \equiv C\left[C_{1}\left[s_{1}, \ldots, s_{n}\right]\right]$ and $t \equiv C\left[C_{2}\left[t_{1}, \ldots, t_{m}\right]\right]$,

(2) $s_{i} \approx s_{j}$ whenever $x_{i} \equiv x_{j}$ for $1 \leq i<j \leq n$,

(3) $t_{i} \equiv \wedge\left\{s_{j} \mid x_{j} \equiv y_{i}\right\}$ for $i=1, \ldots, m$.

We call $C_{1}\left[s_{1}, \ldots, s_{n}\right]$ a marked redex and the relation $\rightarrow_{m}$ is called marked reduction.

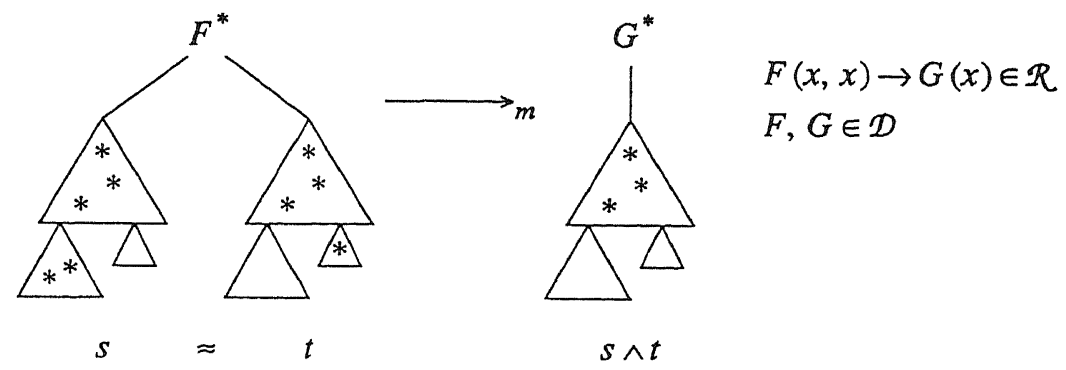

FIGURE 1.

Notice that $\rightarrow_{m}$ coincides with $\rightarrow_{\mathcal{R} \cup \mathcal{R}^{*}}$ whenever $R$ is left-linear.

EXAMPLE 2.4. Consider the CS $(\mathcal{D}, C, \mathcal{R})$ with $\mathcal{D}=\{F, G\}, C=\{S, 0\}$,

$$
R= \begin{cases}F(x, x) & \rightarrow S(x) \\ G(x) & \rightarrow 0\end{cases}
$$

and the reduction sequence $F(G(F(0, S(0))), G(F(0, S(0)))) \rightarrow S(G(F(0, S(0)))) \rightarrow S(0)$. We have $F^{*}\left(G^{*}\left(F^{*}(0, S(0))\right), G^{*}(F(0, S(0)))\right) \rightarrow_{m} S\left(G^{*}(F(0, S(0)))\right) \rightarrow_{m} S(0)$.

The next proposition relates marked reduction to ordinary reduction. In part (2) it is essential that we restrict ourselves to CS's.

PROPOSITION 2.5.

(1) If $s \rightarrow_{m}$ then $e(s) \rightarrow e(t)$.

(2) If $s \rightarrow$ t and $e\left(s^{\prime}\right) \equiv s$ then there exists a term $t^{\prime}$ such that $s^{\prime} \rightarrow_{m} t^{\prime}$ and $e\left(t^{\prime}\right) \equiv t$.

DEFINTTION 2.6. If $t \equiv C\left[t_{1}, \ldots, t_{n}\right]$ such that all defined symbols in $C[, \ldots$,$] are marked$ and every $t_{i}(i=1, \ldots, n)$ is unmarked then we call $t$ a capped term. Furthermore, if $\operatorname{root}\left(t_{i}\right) \in \mathcal{D}$ for $i=1, \ldots, n$ then we write $t \equiv C *\left[t_{1}, \ldots, t_{n}\right] *$.

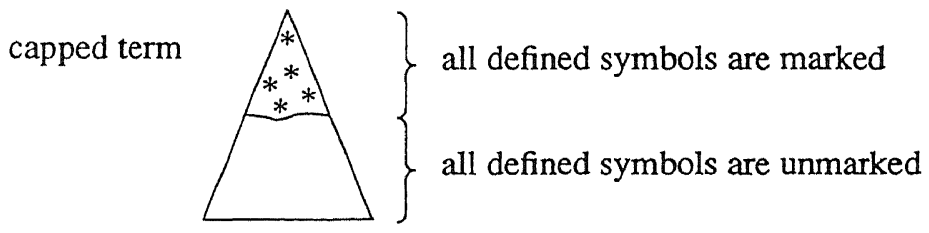

FIGURE 2. 
DEFINTTION 2.7. Let $s \equiv C *\left[s_{1}, \ldots, s_{n}\right] *$ be a capped term.

(1) Suppose $s \rightarrow_{m} t$ by contraction of the marked redex $\Delta$. We write $s \rightarrow_{m}^{i} t$ if $\Delta$ occurs in one of $s_{1}, \ldots, s_{n}$ and we write $s \rightarrow_{m}^{o} t$ otherwise. The relation $\rightarrow_{m}^{i}$ is called inner marked reduction and $\rightarrow_{m}^{o}$ is called outer marked reduction.

(2) We call $s$ inside normalized if it is a normal form with respect to $\rightarrow{ }_{m}^{i}$.

DEFINITION 2.8. Let $t \equiv C *\left[t_{1}, \ldots, t_{n}\right] *$ be a capped term. If $t_{1}, \ldots, t_{n}$ are semi-complete then we define $\psi(t) \equiv C\left[t_{1} \downarrow, \ldots, t_{n} \downarrow\right]$. Notice that $\psi(t)$ is inside normalized.

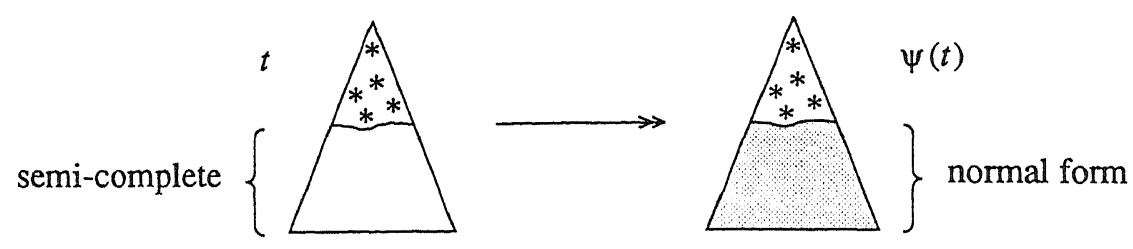

FIGURE 3.

LEMMA 2.9. Let $s$ be a capped term such that $\psi(s)$ is defined.

(1) If $s \rightarrow_{m}^{o} t$ then $\psi(t)$ is defined and $\psi(s) \rightarrow_{m}^{o+} \psi(t)$.

(2) If $s \rightarrow \rightarrow_{m}^{i}$ then $\psi(t)$ is defined and $\psi(s) \equiv \psi(t)$.

(3) If $s$ is a normal form then $\psi(s) \equiv s$.
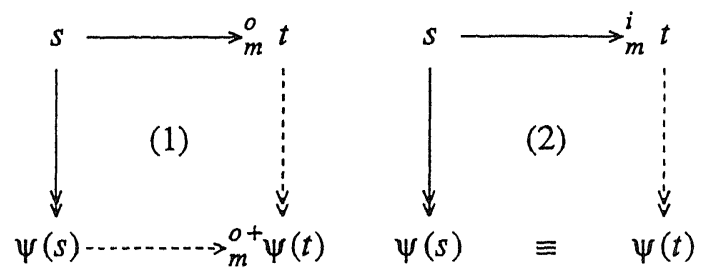

FIGURE 4.

In the remainder of this section we give some further properties of marked reduction which are needed in the next section.

LEMMA 2.10. Let $s$ be a capped term and suppose $s \rightarrow_{m} t$. For every subterm $u$ of $t$ with root $(u) \in \mathcal{D}$ we can find terms $s^{\prime} \subseteq s$ and $t^{\prime} \subseteq t$ such that root $\left(s^{\prime}\right) \in \mathcal{D}, s^{\prime} \rightarrow t^{\prime}$ and $u \subseteq t^{\prime}$.

DEFINTTION 2.11. Let $t$ be a marked term.

(1) The set $\left\{F \in \mathcal{D} \mid F^{*}\right.$ occurs in $\left.t\right\}$ is denoted by $\mathcal{D}^{*}(t)$.

(2) A subset $\mathcal{D}^{\prime}$ of $\mathcal{D}$ is unreachable from $t$ if $\mathcal{D}^{\prime} \cap \mathcal{D}^{*}\left(t^{\prime}\right)=\varnothing$ whenever $t \rightarrow \gg_{m} t^{\prime}$.

DEFINTTION 2.12. Let $\mathcal{D}^{\prime}$ be a subset of $\mathcal{D}$.

(1) A set of pairs $\phi=\left\{\left\langle s_{1}, x_{1}\right\rangle, \ldots,\left\langle s_{n}, x_{n}\right\rangle\right\}$ is a $\mathcal{D}^{\prime}$-replacement if $x_{1}, \ldots, x_{n}$ are mutually distinct variables and $s_{1}, \ldots, s_{n}$ are mutually distinct unmarked terms such that $\operatorname{root}\left(s_{i}\right) \in \mathcal{D}^{\prime}$ for $i=1, \ldots, n$. Let $t \equiv C\left[t_{1}, \ldots, t_{m}\right]$ such that all maximal subterms of $t$ 
with root symbol in $\mathcal{D}^{\prime}$ are displayed. We say that $\phi$ is applicable to $t$ if $x_{1}, \ldots, x_{n}$ do not occur in $t$ and $\left\{t_{1}, \ldots, t_{m}\right\} \subseteq\left\{s_{1}, \ldots, s_{n}\right\}$. In this case we may write $t \equiv C\left[s_{i_{1}}, \ldots, s_{i_{m}}\right]$ with $1 \leq i_{1}, \ldots, i_{m} \leq n$ and we define $\phi(t) \equiv C\left[x_{i_{1}}, \ldots, x_{i_{m}}\right]$.

(2) Let $\phi=\left\{\left\langle s_{1}, x_{1}\right\rangle, \ldots,\left\langle s_{n}, x_{n}\right\rangle\right\}$ be a $\mathcal{D}^{\prime}$-replacement. Suppose $t \equiv C\left[x_{i_{1}}, \ldots, x_{i_{m}}\right]$ such that all occurrences of the variables $x_{1}, \ldots, x_{n}$ in $t$ are displayed. The term $C\left[s_{i_{1}}, \ldots, s_{i_{m}}\right]$ is denoted by $\phi^{-1}(t)$.

PROPOSITION 2.13. Let $\mathcal{D}^{\prime}$ be a subset of $\mathcal{D}$. For every term $t$ there exists a $\mathcal{D}^{\prime}$-replacement $\phi$ which is applicable to $t$.

PROPOSITION 2.14. Let $\phi$ be a $D^{\prime}$-replacement for some $\mathcal{D}^{\prime} \subseteq \mathcal{D}$.

(1) If $s \rightarrow_{m}$ t then $\phi^{-1}(s) \rightarrow_{m} \phi^{-1}(t)$.

(2) If $\phi$ is applicable to then $\phi^{-1}(\phi(t)) \equiv t$.

(3) If $\phi$ is applicable to a capped term then $e(\phi(t)) \equiv \phi(e(t))$.

LEMMA 2.15. Let $s$ be a capped term. Suppose $\mathcal{D}^{\prime} \subseteq \mathcal{D}$ is unreachable from $s$ and $\phi$ is a $\mathcal{D}^{\prime}$ replacement applicable to $s$.

(1) If $s \rightarrow_{m}^{o} t$ then $\phi$ is applicable to $t$ and $\phi(s) \rightarrow_{m}^{o} \phi(t)$.

(2) If $s$ has an infinite $\rightarrow_{m}^{o}$-reduction then $\phi(s)$ has an infinite $\rightarrow_{m}^{o}$-reduction.

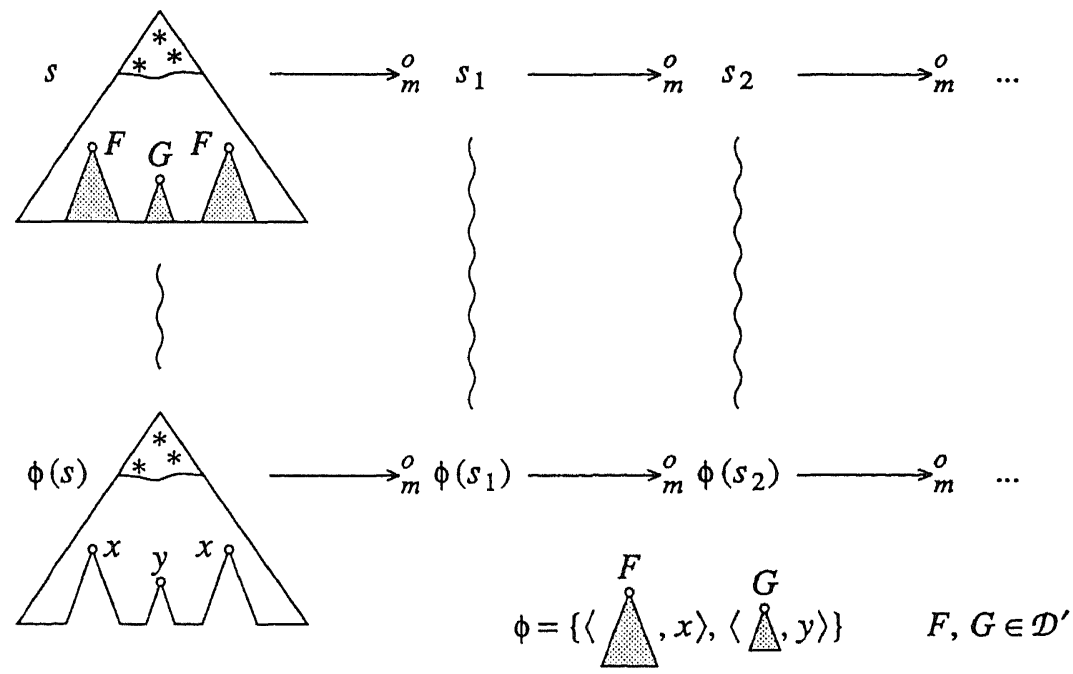

FIGURE 5.

\section{Combinations of Constructor Systems}

In this section we show that both completeness and semi-completeness exhibit the important compositional behaviour expressed in the next definition.

DEFINITION 3.1.

(1) Let $(\mathcal{D}, C, R)$ be a $C S$ and suppose $\mathcal{D}^{\prime} \subseteq \mathcal{D}$. The set $\left\{l \rightarrow r \in \mathscr{R} \mid \operatorname{root}(l) \in \mathcal{D}^{\prime}\right\}$ is 
denoted by $R \mid \mathcal{D}^{\prime}$.

(2) Two CS's $\left(\mathcal{D}_{1}, C_{1}, R_{1}\right)$ and $\left(\mathcal{D}_{2}, C_{2}, R_{2}\right)$ are composable if $\mathcal{D}_{1} \cap C_{2}=\mathcal{D}_{2} \cap C_{1}=\varnothing$ and $R_{1}\left|\mathcal{D}_{2}=R_{2}\right| \mathcal{D}_{1}$. The second requirement is equivalent to the condition that both CS's contain all rewrite rules which 'define' a defined symbol whenever that symbol is shared. The union of pairwise composable CS's $C S_{1}, \ldots, C S_{n}$ is denoted by $C S_{1}+\ldots+C S_{n}$ and we say that $C S_{1}, \ldots, C S_{n}$ is a decomposition of $C S_{1}+\ldots+C S_{n}$.

(3) A property $\mathcal{P}$ of CS's is decomposable if for all pairwise composable CS's $C S_{1}, \ldots, C S_{n}$ with the property $\mathscr{P}$ we have that $C S_{1}+\ldots+C S_{n}$ has the property $\mathscr{P}$.

The counterexample of Toyama against the modularity of strong normalization shows that strong normalization is not a decomposable property of CS's. The following example of Huet [5] shows that also confluence is not decomposable.

EXAMPLE 3.2. Consider the $\operatorname{CS}(\mathcal{D}, C, \mathcal{R})$ with $\mathcal{D}=\{F, C\}, C=\{S, A, B\}$ and

$$
\mathcal{R}= \begin{cases}F(x, x) & \rightarrow A \\ F(x, S(x)) & \rightarrow B \\ C & \rightarrow S(C) .\end{cases}
$$

Let $\mathcal{D}_{1}=\{F\}, C_{1}=C, \mathcal{D}_{2}=\{C\}$ and $C_{2}=\{S\}$. The confluent CS's $\left(\mathcal{D}_{1}, C_{1}, R_{1}\right)$, $\left(\mathcal{D}_{2}, C_{2}, R_{2}\right)$ constitute a decomposition of $(\mathcal{D}, C, R)$, but $(\mathcal{D}, C, R)$ is not confluent since the term $F(C, C)$ can be reduced to the different normal forms $A$ and $B$.

PROPOSITION 3.3. let $\mathcal{P}$ be a property of CS's. The following statements are equivalent:

(1) $P$ is decomposable;

(2) for all composable $C S^{\prime} s C S_{1}$ and $C S_{2}$ with the property $P$ we have that $C S_{1}+C S_{2}$ has the property $P$.

PROOF. Straightforward.

LEMMA 3.4. Local confluence is decomposable.

PROOF. Let $\left(\mathcal{D}_{1}, \mathcal{C}_{1}, \mathcal{R}_{1}\right)$ and $\left(\mathcal{D}_{2}, C_{2}, \mathcal{R}_{2}\right)$ be locally confluent and decomposable CS's. We have to show that their union $(\mathcal{D}, \mathcal{C}, \mathcal{R})$ is locally confluent. According to the Critical Pair Lemma it sufficient to show that every critical pair of $(\mathcal{D}, \mathcal{C}, \mathcal{R})$ is convergent. If $\langle s, t\rangle$ is a critical pair of $(\mathcal{D}, \mathcal{C}, \mathcal{R})$ then there exist rewrite rules $l_{1} \rightarrow r_{1}, l_{2} \rightarrow r_{2} \in \mathcal{R}$ and a substitution $\sigma$ such that $l_{1}^{\sigma} \equiv l_{2}^{\sigma}, s \equiv r_{1}^{\sigma}$ and $t \equiv r_{2}^{\sigma}$. Choose $k \in\{1,2\}$ such that $\operatorname{root}\left(l_{1}\right)=\operatorname{root}\left(l_{2}\right) \in \mathcal{D}_{k}$. We have $l_{1} \rightarrow r_{1}, l_{2} \rightarrow r_{2} \in \mathcal{R}_{k}$ and because $\left(\mathcal{D}_{k}, C_{k}, R_{k}\right)$ is locally confluent $\langle s, t\rangle$ is $\mathcal{R}_{k}$-convergent and hence also $\mathcal{R}$-convergent.

THEOREM 3.5. Completeness is decomposable.

PROOF. Let $\left(\mathcal{D}_{1}, \mathcal{C}_{1}, \mathcal{R}_{1}\right)$ and $\left(\mathcal{D}_{2}, \mathcal{C}_{2}, \mathcal{R}_{2}\right)$ be complete and composable CS's. From Lemma 3.4 we obtain the local confluence of their union $(\mathcal{D}, C, R)$. According to Newman's Lemma it suffices to show the strong normalization of $(\mathcal{D}, C, R)$. This will be established by induction on the structure of terms $t \in \mathcal{T}(\mathcal{D}, C, \mathcal{V})$. If $t$ is a variable or a constructor constant then $t$ is a normal form. If $t$ is a defined constant then $t$ belongs to some $\mathcal{D}_{k}$ and because $\left(\mathcal{D}_{k}, C_{k}, \mathcal{R}_{k}\right)$ is strongly normalizing $t$ cannot have an infinite reduction. For the induction step, let $t \equiv F\left(t_{1}, \ldots, t_{n}\right)$ such that $t_{1}, \ldots, t_{n}$ are strongly normalizing (and hence complete). If $F$ is a constructor then $t$ clearly is strongly normalizing. So assume that $F \in \mathcal{D}$. If $t$ is not 
strongly normalizing then there exists an infinite reduction sequence

$$
t \equiv s_{1} \rightarrow s_{2} \rightarrow s_{3} \rightarrow \ldots
$$

Let $t^{\prime} \equiv F^{*}\left(t_{1}, \ldots, t_{n}\right)$. According to Proposition 2.5 we can find terms $s_{i}^{\prime}$ with $e\left(s_{i}^{\prime}\right) \equiv s_{i}$ such that

$$
t^{\prime} \equiv s_{1}^{\prime} \rightarrow_{m} s_{2}^{\prime} \rightarrow_{m} s_{3}^{\prime} \rightarrow_{m} \ldots .
$$

Using Lemma 2.10 and the assumption that $t_{1}, \ldots, t_{n}$ are strongly normalizing, it is not difficult to show that sequence (2) contains infinitely many $\rightarrow_{m}^{o}$-steps. According to Lemma 2.9 we can transform sequence (2) into the marked reduction sequence

$$
\psi\left(t^{\prime}\right) \equiv \psi\left(s_{1}^{\prime}\right) \rightarrow_{m}^{o} \Psi\left(s_{2}^{\prime}\right) \rightarrow_{m}^{o} \psi\left(s_{3}^{\prime}\right) \rightarrow_{m}^{o} \ldots
$$

which contains infinitely many steps. Choose $k \in\{1,2\}$ such that $F \in \mathcal{D}_{k}$ and let $\mathcal{D}^{\prime}=\mathcal{D}-\mathcal{D}_{k}$. It is easy to show that $\mathcal{D}^{\prime}$ is unreachable from $\psi\left(t^{\prime}\right)$. From Proposition 2.13 we obtain a $\mathcal{D}^{\prime}$-replacement $\phi$ which is applicable to $\psi\left(t^{\prime}\right)$. By Lemma $2.15(2)$ the term $\phi\left(\psi\left(t^{\prime}\right)\right)$ has an infinite $\rightarrow_{m}^{o}$-reduction sequence

$$
\phi\left(\psi\left(t^{\prime}\right)\right) \equiv \phi\left(\Psi\left(s_{1}^{\prime}\right)\right) \rightarrow_{m}^{o} \phi\left(\psi\left(s_{2}^{\prime}\right)\right) \rightarrow_{m}^{o} \phi\left(\Psi\left(s_{3}^{\prime}\right)\right) \rightarrow_{m}^{o} \ldots .
$$

If we erase all markers in this sequence we obtain an infinite reduction sequence starting from the term $e\left(\phi\left(\psi\left(t^{\prime}\right)\right)\right)$. This contradicts the strong normalization of the $\operatorname{CS}\left(\mathcal{D}_{k}, C_{k}, R_{k}\right)$.

COROLLARY 3.6. Completeness is a modular property of CS's.

COROLLARY 3.7. The union of complete CS's which do not share defined symbols is complete.

\begin{tabular}{|c|c|c|c|c|c|c|c|}
\hline$r_{1}$ & $0+x$ & $\rightarrow$ & $x$ & $r_{8}$ & $x<0$ & $\rightarrow$ & false \\
\hline$r_{2}$ & $S(x)+y$ & $\rightarrow$ & $S(x+y)$ & $r_{9}$ & $0<S(x)$ & $\rightarrow$ & true \\
\hline$r_{3}$ & $0 \times x$ & $\rightarrow$ & 0 & $r_{10}$ & $S(x)<S(y)$ & $\rightarrow$ & $x<y$ \\
\hline$r_{4}$ & $S(x) \times y$ & $\rightarrow$ & $x \times y+y$ & $r_{11}$ & true $\wedge$ false & $\rightarrow$ & false \\
\hline$r_{5}$ & $f i b(0)$ & $\rightarrow$ & $S(0)$ & $r_{12}$ & false $\wedge$ true & $\rightarrow$ & false \\
\hline$r_{6}$ & $f i b(S(0))$ & $\rightarrow$ & $S(0)$ & $r_{13}$ & $x \wedge x$ & $\rightarrow$ & $x$ \\
\hline$r_{7}$ & $f i b(S(S(x)))$ & $\rightarrow$ & $f i b(S(x))+f i b(x)$ & & & & \\
\hline
\end{tabular}

We now consider a more challenging situation in which Theorem 3.5 can be applied.

EXAMPLE 3.8. Consider the $\operatorname{CS}(\mathcal{D}, \mathcal{C}, \mathcal{R})$ with $\mathcal{D}=\{+, \times, f i b,<, \wedge\}, \mathcal{C}=\{0, S$, true, false $\}$ and rewrite rules

Consider the decomposition $\left(\mathcal{D}_{i}, \mathcal{C}_{i}, \mathcal{R}_{i}\right)_{i=1}^{4}$ defined as follows:

\begin{tabular}{l|lll}
$i$ & $\mathcal{D}_{i}$ & $C_{i}$ & $R_{i}$ \\
\hline 1 & $+\times$ & $0 S$ & $r_{1} r_{2} r_{3} r_{4}$ \\
2 & + fib & $0 S$ & $r_{1} r_{2} r_{5} r_{6} r_{7}$ \\
3 & $<$ & $0 S$ true false & $r_{8} r_{9} r_{10}$ \\
4 & $\wedge$ & true false & $r_{11} r_{12} r_{13}$
\end{tabular}

Routine arguments show that every $\left(\mathcal{D}_{i}, C_{i}, R_{i}\right)$ is complete. Theorem 3.5 yields the completeness of $(\mathcal{D}, C, \mathcal{R})$. 
The proof of the decomposability of semi-completeness is comparable to the proof of Theorem 3.5. First we show the decomposability of weak normalization.

LEMMA 3.9. Weak normalization is decomposable.

PRoOF. Suppose $\left(\mathcal{D}_{1}, \mathcal{C}_{1}, R_{1}\right)$ and $\left(\mathcal{D}_{2}, \mathcal{C}_{2}, R_{2}\right)$ are weakly normalizing and composable CS's and let $(\mathcal{D}, \mathcal{C}, \mathcal{R})=\left(\mathcal{D}_{1}, C_{1}, R_{1}\right)+\left(\mathcal{D}_{2}, C_{2}, R_{2}\right)$. We will show by induction on the structure of $t$ that every term $t \in \mathcal{T}(\mathcal{D}, \mathcal{C}, \mathcal{V})$ has a normal form. The case $t \in \mathcal{D} \cup \mathcal{C} \cup \mathcal{V}$ is easy. Suppose $t \equiv F\left(t_{1}, \ldots, t_{n}\right)$ and $t_{1}, \ldots, t_{n}$ are weakly normalizing. Let $s_{i}$ be a normal form of $t_{i}$ for $i=1, \ldots, n$ and define $t^{\prime} \equiv F\left(s_{1}, \ldots, s_{n}\right)$. If $F \in C$ then $t^{\prime}$ is a normal form of $t$. If $F \in \mathcal{D}$ then there exists a $k \in\{1,2\}$ such that $F \in \mathcal{D}_{k}$. Let $\mathcal{D}^{\prime}=\mathcal{D}-\mathcal{D}_{k}$. From Proposition 2.13 we obtain a $\mathcal{D}^{\prime}$-replacement $\phi$ which is applicable to $t^{\prime}$. Since $\left(\mathcal{D}_{k}, \mathcal{C}_{k}, \mathcal{R}_{k}\right)$ is weakly normalizing, the term $\phi\left(t^{\prime}\right)$ has a normal form, say $t^{\prime \prime}$. Using Proposition 2.14 we obtain $t \rightarrow t^{\prime} \equiv \phi^{-1}\left(\phi\left(t^{\prime}\right)\right) \rightarrow \phi^{-1}\left(t^{\prime \prime}\right)$. It is easy to show that $\phi^{-1}\left(t^{\prime \prime}\right)$ is a normal form.

THEOREM 3.10. Semi-completeness is decomposable.

PROOF. Let $\left(\mathcal{D}_{1}, \mathcal{C}_{1}, \mathcal{R}_{1}\right)$ and $\left(\mathcal{D}_{2}, \mathcal{C}_{2}, \mathcal{R}_{2}\right)$ be semi-complete and composable CS's. From Lemma 3.9 we obtain the weak normalization of their union $(\mathcal{D}, \mathcal{C}, \mathcal{R})$. Hence it is sufficient to show that every term $t \in \mathcal{T}(\mathcal{D}, C, \mathcal{V})$ has at most one normal form. We use induction on the structure of $t$. The case $t \in \mathcal{D} \cup C \cup \mathcal{V}$ is easy. Suppose $t \equiv F\left(t_{1}, \ldots, t_{n}\right)$ such that every $t_{i}$ is semi-complete. If $F \in C$ then $F\left(t_{1} \downarrow, \ldots, t_{n} \downarrow\right)$ is the unique normal form of $t$. Suppose $F \in \mathcal{D}$ and let $t^{\prime} \equiv F^{*}\left(t_{1}, \ldots, t_{n}\right)$. Define $\mathcal{D}_{k}, \mathcal{D}^{\prime}$ and $\phi$ as in the proof of Theorem 3.5. First we show that if $t$ has a normal form $n$ then $\phi\left(e\left(\psi\left(t^{\prime}\right)\right)\right) \rightarrow \phi(n)$. With help of Proposition 2.5 and Lemma 2.9 we obtain a normal form $n^{\prime}$ such that $\psi\left(t^{\prime}\right) \rightarrow_{m}^{o} n^{\prime}$ and $e\left(n^{\prime}\right) \equiv n$. Repeated application of Lemma $2.15(1)$ yields $\phi\left(\psi\left(t^{\prime}\right)\right) \rightarrow_{m}^{o} \phi\left(n^{\prime}\right)$. Erasing all markers in this sequences gives us $e\left(\phi\left(\psi\left(t^{\prime}\right)\right)\right) \rightarrow e\left(\phi\left(n^{\prime}\right)\right)$ and from Proposition 2.14(3) we obtain $e\left(\phi\left(\psi\left(t^{\prime}\right)\right)\right) \equiv \phi\left(e\left(\psi\left(t^{\prime}\right)\right)\right)$ and $e\left(\phi\left(n^{\prime}\right)\right) \equiv \phi\left(e\left(n^{\prime}\right)\right) \equiv \phi(n)$. Now suppose that $t$ has normal forms $n_{1}$ and $n_{2}$. From the above discussion we learn that $\phi\left(n_{1}\right) \lll \phi\left(e\left(\psi\left(t^{\prime}\right)\right)\right) \rightarrow \phi\left(n_{2}\right)$. Notice that $\phi\left(n_{1}\right)$ and $\phi\left(n_{2}\right)$ are normal forms. We obtain $\phi\left(n_{1}\right) \equiv \phi\left(n_{2}\right)$ from the semicompleteness of $\left(\mathcal{D}_{k}, \mathcal{C}_{k}, \mathcal{R}_{k}\right)$. Hence $n_{1} \equiv \phi^{-1}\left(\phi\left(n_{1}\right)\right) \equiv \phi^{-1}\left(\phi\left(n_{2}\right)\right) \equiv n_{2}$ by Proposition 2.14(2).

COROLLARY 3.11. The union of semi-complete CS's which do not share defined symbols is semi-complete.

\section{References}

1. N. Dershowitz, Termination of Linear Rewriting Systems (preliminary version), Proceedings of the 8th International Colloquium on Automata, Languages and Programming, Acre, Lecture Notes in Computer Science 115, pp. 448-458, 1981.

2. N. Dershowitz and J.-P. Jouannaud, Rewrite Systems, in: Handbook of Theoretical Computer Science, Vol. B (ed. J. van Leeuwen), North-Holland, 1990.

3. K. Drosten, Termersetzungssysteme, Informatik-Fachberichte 210, Springer, 1989 (in German).

4. A. Geser, Relative Termination, Ph.D. thesis, University of Passau, 1990.

5. G. Huet, Confluent Reductions: Abstract Properties and Applications to Term Rewriting Systems, Journal of the ACM 27(4), pp. 797-821, 1980.

6. J.W. Klop, Term Rewriting Systems, to appear in: Handbook of Logic in Computer 
Science, Vol. I (eds. S. Abramsky, D. Gabbay and T. Maibaum), Oxford University Press, 1991.

7. M. Kurihara and I. Kaji, Modular Term Rewriting Systems: Termination, Confluence and Strategies, Report, Hokkaido University, Sapporo, 1988. (Abridged version: Modular Term Rewriting Systems and the Termination, Information Processing Letters 34, pp. 1-4, 1990.)

8. M. Kurihara and A. Ohuchi, Modularity of Simple Termination of Term Rewriting Systems, Journal of IPS Japan 31(5), pp. 633-642, 1990.

9. M. Kurihara and A. Ohuchi, Modularity of Simple Termination of Term Rewriting Systems with Shared Constructors, Report SF-36, Hokkaido University, Sapporo, 1990.

10. A. Middeldorp, Modular Aspects of Properties of Term Rewriting Systems Related to Normal Forms, Proceedings of the 3rd International Conference on Rewriting Techniques and Applications, Chapel Hill, Lecture Notes in Computer Science 355, pp. 263-277, 1989. (Full version: Report IR-164, Vrije Universiteit, Amsterdam, 1988.)

11. A. Middeldorp, A Sufficient Condition for the Termination of the Direct Sum of Term Rewriting Systems, Proceedings of the 4th IEEE Symposium on Logic in Computer Science, Pacific Grove, pp. 396-401, 1989.

12. A. Middeldorp, Confluence of the Disjoint Union of Conditional Term Rewriting Systems, Report CS-R8944, Centre for Mathematics and Computer Science, Amsterdam, 1989. (To appear in: Proceedings of the 2nd International Workshop on Conditional and Typed Rewriting Systems, Montreal, 1990.)

13. A. Middeldorp, Termination of Disjoint Unions of Conditional Term Rewriting Systems, Report CS-R8959, Centre for Mathematics and Computer Science, Amsterdam, 1989.

14. A. Middeldorp, Unique Normal Forms for Disjoint Unions of Conditional Term Rewriting Systems, Report CS-R9003, Centre for Mathematics and Computer Science, Amsterdam, 1990.

15. A. Middeldorp, Modular Properties of Term Rewriting Systems, Ph.D. thesis, Vrije Universiteit, Amsterdam, 1990.

16. M.H.A. Newman, On Theories with a Combinatorial Definition of Equivalence, Annals of Mathematics 43(2), pp. 223-243, 1942.

17. M.J. O'Donnell, Equational Logic as a Programming Language, The MIT Press, 1985.

18. M. Rusinowitch, On Termination of the Direct Sum of Term Rewriting Systems, Information Processing Letters 26, pp. 65-70, 1987.

19. Y. Toyama, On the Church-Rosser Property for the Direct Sum of Term Rewriting Systems, Journal of the ACM 34(1), pp. 128-143, 1987.

20. Y. Toyama, Counterexamples to Termination for the Direct Sum of Term Rewriting Systems, Information Processing Letters 25, pp. 141-143, 1987.

21. Y. Toyama, Commutativity of Term Rewriting Systems, in: Programming of Future Generation Computer II (eds. K. Fuchi and L. Kott), North-Holland, pp. 393-407, 1988.

22. Y. Toyama, J.W. Klop and H.P. Barendregt, Termination for the Direct Sum of LeftLinear Term Rewriting Systems, Proceedings of the 3rd International Conference on Rewriting Techniques and Applications, Chapel Hill, Lecture Notes in Computer Science 355, pp. 477-491, 1989. 